Bond University

Research Repository

\title{
Do we really know what they were testing? Incomplete reporting of interventions in randomised trials of upper limb therapies in unilateral cerebral palsy
}

\author{
Sakzewski, Leanne; Reedman, Sarah; Hoffmann, Tammy
}

Published in:

Research in Developmental Disabilities

DOI:

10.1016/j.ridd .2016 .09 .018

Licence:

CC BY-NC-ND

Link to output in Bond University research repository.

Recommended citation(APA):

Sakzewski, L., Reedman, S., \& Hoffmann, T. (2016). Do we really know what they were testing? Incomplete reporting of interventions in randomised trials of upper limb therapies in unilateral cerebral palsy. Research in Developmental Disabilities, 59, 417-427. https://doi.org/10.1016/j.ridd.2016.09.018

\section{General rights}

Copyright and moral rights for the publications made accessible in the public portal are retained by the authors and/or other copyright owners and it is a condition of accessing publications that users recognise and abide by the legal requirements associated with these rights.

For more information, or if you believe that this document breaches copyright, please contact the Bond University research repository coordinator. 
Running heading: Intervention descriptions in cerebral palsy research

Title: Do we really know what they were testing? Incomplete reporting of interventions in randomised trials of upper limb therapies in unilateral cerebral palsy

\section{Leanne Sakzewski, $\mathbf{P h D}^{1}$}

Sarah Reedman, BPhty ${ }^{1}$

Tammy Hoffmann, $\mathrm{PhD}^{2}$

${ }^{1}$ Queensland Cerebral Palsy and Rehabilitation Research Centre, School of Medicine, The University of Queensland, Brisbane, Australia

${ }^{2}$ Centre for Research in Evidence-Based Practice, Faculty of Health Sciences and Medicine, Bond University, Gold Coast, Australia

\section{Corresponding author}

Leanne Sakzewski

Queensland Cerebral Palsy and Rehabilitation Research Centre

The University of Queensland

Centre for Children's Health Research,

62 Graham Street

South Brisbane, Queensland, 4101

Australia

Phone: $\quad+1(617) 30697345$

Email: $\quad \underline{\text { 1.sakzewski1@uq.edu.au }}$

Conflict of Interest: The authors have no conflict of interests to declare 


\section{ABSTRACT}

Background: Incomplete reporting of components of interventions limits uptake of evidence into clinical practice.

Aims: To evaluate the completeness of reporting of research and control interventions in randomised trials of upper limb therapies for children with unilateral cerebral palsy.

Methods and procedures: Sixty randomized trials were included, encompassing 60 research and 68 control interventions. Using the 12-item Template for Intervention Description and Replication (TIDieR) checklist, two reviewers independently rated intervention and control descriptions.

Outcomes and results: When using 50\% of studies as the benchmark, five of the 12 TIDieR items for the research intervention, eight of the 12 items for the control intervention and 11 of 12 items for "usual care" interventions were inadequately reported. Procedures used to deliver the research intervention were adequately reported for $63 \%$ of studies. Materials were used in $94 \%$ of research interventions, yet only $27 \%$ provided details to access/replicate materials. Training materials for interventionists were used in $38 \%$ of trials, $10(17 \%)$ had procedure manuals, yet only 3 reported details to access materials. The location where the research intervention was provided was detailed in $65 \%$ of studies. Reporting of all items was poorer for the control intervention.

Conclusions: No study adequately reported all elements on the TIDieR checklist. Details crucial for replication of interventions and interpretation of results were missing. Authors, reviewers, and editors all have a responsibility to improve the quality of intervention reporting in published trials. The TIDieR guide is a potential solution, helping to structure accounts of interventions.

\section{What this study adds:}

This is the first study 
- This is the first study to evaluate the completeness of reporting of intervention components in complex cerebral palsy interventions

- This is the first study to rate completeness of reporting of control interventions

- This study provides information to increase awareness of editors, reviewers and authors of the importance of complete reporting of intervention components in cerebral palsy rehabilitation.

Key Words: cerebral palsy, upper limb, rehabilitation, randomised trials; intervention description

\section{Abbreviations:}

CIMT Constraint induced movement therapy

CP Cerebral Palsy

TIDieR Template for Intervention Description and Replication 


\section{Introduction}

In the last decade, there has been a rapid increase in evidence from clinical trials for a variety of upper limb therapies for children with unilateral cerebral palsy (Novak, McIntyre, Morgan, Campbell, Dark, Morton et al., 2013; Sakzewski, Ziviani, \& Boyd, 2014). Despite there being over 45 published randomized trials, there is a lag in the uptake of this evidence into clinical practice. In particular, the uptake of contemporary motor learning based approaches such as constraint induced movement therapy, intensive bimanual therapy and hybrid models combining the two approaches has been slow (McConnell, Johnston, \& Kerr, 2012; Schertz \& Gordon, 2008).

Incomplete description of interventions in randomized trials is one barrier to evidence uptake, yet one that is remediable. Incomplete descriptions of interventions limit clinicians' ability to reliably use those that have proven efficacy. There are also implications for researchers who seek to replicate and extend on previous research findings, and service managers, policy makers and consumers who endeavour to ensure that current evidence is effectively incorporated into contemporary service delivery. Lack of detail for control interventions in randomized trials further impacts interpretation of the magnitude of treatment effect of the therapies being evaluated.

Inadequate reporting of interventions is considered part of the final stage of waste that can occur across the continuum from research generation to publication (Glasziou, Altman, Bossuyt, Boutron, Clarke, Julious et al., 2014). The extent of inadequate reporting of interventions has been investigated in a number of studies, with between 50 to 60 percent of papers missing essential elements in the description of the interventions (Glasziou, Meats, Heneghan, \& Shepperd, 2008; Schroter, Glasziou, \& Heneghan, 2012). Reporting of nonpharmacological interventions is typically worse than pharmacological interventions (Douet, Milne, Anstee, Habens, Young, \& Wright, 2014; Glasziou et al., 2008). In a cross-sectional 
analysis of 137 non-pharmacological interventions, only 39\% were described adequately in the primary paper, protocols or related websites (Hoffmann, Erueti, \& Glasziou, 2013). The completeness of description of control interventions in randomized trials has not been explored in any previous studies.

To help authors of evaluative studies provide complete descriptions of interventions, the Template for Intervention Description and Replication (TIDieR) guide and checklist was recently published (Hoffmann, Glasziou, Boutron, Milne, Perera, Moher et al., 2014b).

TIDieR is an extension of Item 5 of the Consolidated Standards of Reporting Trials (CONSORT) 2010 Statement (Moher, Hopewell, Schulz, Montori, Gotzsche, Devereaux et al., 2010) and Item 11 of a guide for reporting trial protocols (SPIRIT Standard Protocol Items: Recommendations for Intervention Trials) (Chan, Tetzlaff, Altman, Laupacis, Gotzsche, Krleza-Jeric et al., 2013).

This analysis of trials from a systematic review (Sakzewski et al., 2014) and subsequent publications of non-surgical upper limb therapies for children with unilateral cerebral palsy aimed to evaluate the completeness of reporting of both intervention and control therapies using the TIDieR checklist.

\section{Method}

\subsection{Search strategy and trial selection}

Forty-two studies reporting 13 upper limb therapy approaches identified in a recently published systematic review (Sakzewski et al., 2014) were included. This systematic review included randomised controlled trials of non-surgical upper limb interventions for children aged 0 to 18 years with unilateral cerebral palsy to improve upper limb capacity and performance, individualised outcomes and self-care skills. A subsequent search following the same method and inclusion criteria used in the systematic review (Sakzewski et al., 2014) was conducted in May 2015 to ensure that any new trials were also included in this study. 
For each included study, reference lists were scrutinised and citation tracking used to identify other related publications, trial protocols, additional appendices, online supplementary materials, or relevant web-based resources. Study protocols of ongoing trials were not included unless the primary outcome paper of the study had been published.

\subsection{Rating of research intervention and control descriptions}

The TIDieR checklist comprises 12 items to guide reporting on the rationale underpinning the intervention, materials and procedures, intervention providers and location, individualization or tailoring, measurement and reporting of treatment adherence/fidelity, and any intervention modifications between the protocol and trial or during the trial itself (Table 1) (Hoffmann et al., 2014b). The TIDieR checklist and guide was used to rate the completeness of descriptions of interventions and control therapies of included studies. Descriptions of interventions and control conditions were rated separately. Control interventions were either a dose matched alternative therapy to the research intervention, or were reported as "usual care", "traditional rehabilitation", "standard care” or similar descriptor. When the study compared two dose matched interventions, the categorisation of the intervention as being research or control reflected the specific aims and directional hypotheses articulated in the study. In studies where there was more than one control condition, each was rated separately on the TIDieR checklist. For trials where the intervention also contained a pharmacological component, such as Botulinum Toxin A, only the non-pharmacological rehabilitation/therapy component of the intervention was rated.

Each TIDieR item was rated as "yes", indicating the item had been adequately described or "no" indicating inadequate or incomplete reporting. Many of the items in the TIDieR checklist have a number of separate components. For example: Item 3 "materials" includes both the materials used in the delivery of the intervention and any materials used to train intervention providers. Such items were reported as an overall score, however where 
applicable, elements of the item were recorded so to further understand inadequacies of reporting. As a benchmark to summarise and report findings of the TIDieR ratings, items will be described based on whether they have been reported adequately in more or less than $50 \%$ of studies.

Two researchers independently rated included studies. To maximise consistency between raters, each researcher independently piloted the checklist on five studies and then met to discuss any differences in interpretation of items. The researchers then completed rating of the remaining studies and met to discuss ratings. Disagreements in ratings were discussed until consensus was reached or referred to a third rater $(\mathrm{TH})$ when unable to be resolved. Ratings of trials and study protocols were reported together so that final ratings for intervention and control groups in each research study were determined from all sources of available information (trial reports, study protocols, and any supplementary information available).

\subsection{Analysis}

Data were entered in Excel and analysed descriptively. Ratings for research and control interventions are reported separately. The control interventions described as "usual care" were reported as a separate subgroup of the control interventions.

\section{Results}

Forty-two randomized trials were included in the original systematic review (Sakzewski et al., 2014) and a further 18 were identified from the subsequent search (Abd ElKafy, Elshemy, \& Alghamdi, 2014; Bleyenheuft, Arnould, Brandao, Bleyenheuft, \& Gordon, 2014; Brandao, Ferre, Kuo, Rameckers, Bleyenheuft, Hung et al., 2013; Chiu, Ada, \& Lee, 2014; Deppe, Thuemmler, Fleischer, Berger, Meyer, \& Wiedemann, 2013; Dong \& Fong, 2014; Ferrari, Maoret, Muzzini, Alboresi, Lombardi, Sgandurra et al., 2014; Gelkop, Burshtein, Lahav, Brezner, Al-Oraibi, Ferre et al., 2014; Gilliaux, Renders, Dispa, Holvoet, 
Sapin, Dehez et al., 2015; James, Ziviani, Ware, \& Boyd, 2015; Kaya Kara, Atasavun Uysal, Turker, Karayazgan, Gunel, \& Baltaci, 2015; Klingels, Feys, Molenaers, Verbeke, Van Daele, Hoskens et al., 2013; Koman, Smith, Williams, Richardson, Naughton, Griffin et al., 2013; Lidman, Nachemson, Peny-Dahlstrand, \& Himmelmann, 2015; Sakzewski, Miller, Ziviani, Abbott, Rose, Macdonell et al., 2015; Sgandurra, Ferrari, Cossu, Guzzetta, Fogassi, \& Cioni, 2013; Yu, Kang, \& Jung, 2012; Zoccolillo, Morelli, Cincotti, Muzzioli, Gobbetti, Paolucci et al., 2015). In total, 60 randomized trials (the outcomes of which were reported in 71 papers), three feasibility studies informing a randomized trial (Novak, Cusick, \& Lowe, 2007; Wallen, Ziviani, Herbert, Evans, \& Novak, 2009; Wallen, O'Flaherty S, \& Waugh, 2004), and 12 supporting papers reporting the study protocols or development of the intervention (Aarts, van Hartingsveldt, Anderson, van den Tillaar, van der Burg, \& Geurts, 2012; Bleyenheuft \& Gordon, 2014; Boyd, Sakzewski, Ziviani, Abbott, Badawy, Gilmore et al., 2010; Boyd, Mitchell, James, Ziviani, Sakzewski, Smith et al., 2013; Boyd, Ziviani, Sakzewski, Miller, Bowden, Cunnington et al., 2013; Charles \& Gordon, 2006; Eliasson, Krumlinde-Sundholm, Shaw, \& Wang, 2005; Facchin, Rosa-Rizzotto, Turconi, Pagliano, Fazzi, Stortini et al., 2009; Gordon, Charles, \& Wolf, 2005; Hoare, Imms, Rawicki, \& Carey, 2010; Law, Darrah, Pollock, Rosenbaum, Russell, Walter et al., 2007; Sgandurra, Sicola, Di Pietro, Burzi, Filippi, Parente et al., 2011) were included, encompassing 60 research and 68 control interventions. Upper limb interventions included constraint induced movement therapy (CIMT); Hand-Arm Bimanual Intensive Therapy; Botulinum toxin A and occupational therapy; splinting; action observation training; context-focused intervention; mirror therapy; neurodevelopmental therapy; occupational therapy home programs; acupuncture and occupational therapy; kinesiotape; sensory cuing; virtual reality/robotics; and forced use therapy (Table 2).

Items three to nine on the TIDier checklist reflect core elements necessary for 
replication of the intervention. No study adequately reported all of these seven core elements. The percentage of TIDieR items adequately described for the research and control interventions is depicted in Figure 1. The TIDieR items are described in greater detail and examples of good reporting from the included studies are summarized in Table 1.

\subsection{Research Interventions}

The first two items on the TIDieR checklist (brief name Item 1, and rationale Item 2) were the most consistently reported. All studies provided a name and brief description of the intervention being investigated. The rationale underpinning the research intervention was described in $56(93 \%)$ of the studies.

Crucial details about the number of times the intervention was provided, over what length of time, scheduling and duration of sessions was described for $48(80 \%)$ of research interventions (Dose Item 8). The most common missing detail was the duration of therapy sessions.

The mode of therapy delivery (Item 6 "How", e.g. face to face, and whether individual or group-based) was adequately reported in $46(77 \%)$ of research interventions. Delivery of individual therapy rather than group-delivered therapy was often implied, but not explicitly stated. Nine studies $(15 \%)$ reported using group-delivered therapy, with eight (13\%) specifying the group size and child to therapist ratio.

Individualization (tailoring) of the research intervention (Item 9) was mentioned in 46 (77\%) of research interventions, most commonly reflecting the identification and targeting of individual client goals in therapy. However, decision points or guidelines for progression or adaptation of the intervention based on individual participants' response to therapy were described by a few studies (Charles, Wolf, Schneider, \& Gordon, 2006; Elvrum, Braendvik, Saether, Lamvik, Vereijken, \& Roeleveld, 2012; Gordon, Schneider, Chinnan, \& Charles, 2007; Gordon, Hung, Brandao, Ferre, Kuo, Friel et al., 2011). One study of resistance 
training and Botulinum Toxin injections described the grading of the program: "Once the participants were able to perform three sets of ten repetitions in an exercise, intensity was build up progressively on an individualized basis by increasing the weights by $0.25-0.5 \mathrm{~kg}$." (Elvrum et al., 2012). Thirty-nine (65\%) of studies adequately reported the procedure (Item 4) for the research intervention, detailing the activities and/or processes used.

The location of where the intervention occurred (e.g. home, outpatient clinic, community leisure facility - Item 7) was described for 39 (65\%) of research interventions. Details about how intervention fidelity (Items 11 and 12) was measured and reported or any strategies to monitor or maintain it were described in less than $50 \%$ of the studies. Provision of the intervention by more than one therapist/provider is mentioned in $60 \%$ of the trials, which highlights the need for ascertaining fidelity of intervention across interventionists. Of the trials that reported measuring intervention fidelity, methods included: therapist logs detailing the intervention provided; home practice logs describing the amount, frequency and/or type of home practice; or videoed treatment sessions that were later reviewed by the researchers.

Overall, 23 (38\%) of trials reported adequate details on the provider of the research intervention (Item 5) including their profession and either described their expertise, assessed their competence or provided additional therapist training. When the components of this item are considered, $51(85 \%)$ of studies identified the profession of the intervention provider, their expertise (9 studies - 15\%) and $17(28 \%)$ reported providing further interventionspecific training although minimal detail explained the structure, content or method of training.

Less than $20 \%$ of studies adequately reported materials used in the interventions and/or provider training (Item 3). Materials used in the interventions included but were not limited to, written home programs, home program practice logs, splints/mitts/casts or slings, 
lists of home practice activities, videos and written instructions. Most 56 (93\%) trials reported that materials were part of the research intervention, yet only $16(27 \%)$ trials provided sufficient details to either access or replicate all the materials used in the intervention. For example, restraints were not described in enough detail in 13 (46\%) of 28 constraint induced movement therapy and forced use studies to allow replication. An example of inadequate reporting is "participants in the constraint-induced therapy group were required to wear an elastic bandage and restraint glove that limited their wrist and individual finger movement” (Hsin, Chen, Lin, Kang, Chen, \& Chen, 2012). In contrast, in another trial a more detailed description and supporting photograph provided sufficient detail to allow replication: "A comfortable neoprene (wet suit material) glove was worn on the hand of the non-affected upper limb.... The neoprene glove, with palmar thermoplastic insert over the fingers and thumb to prevent grasp, allowed the child to use the hand as an effective assist in bilateral activities, but did not allow active grasp of objects (see Figure 2.)" (Hoare et al., 2010). Home programs were provided in 31 (52\%) of studies however only 7 (23\%) provided adequate description to replicate the programs or details on how to obtain the program from authors. Home programs were variously described as lists of activities, manual of exercises (Klingels et al., 2013), home program with neurodevelopmental activities (Law, Cadman, Rosenbaum, Walter, Russell, \& Dematteo, 1991; Law, Russell, Pollock, Rosenbaum, Walter, \& King, 1997) or written lists of training tasks (Taub, Griffin, Uswatte, Gammons, Nick, \& Law, 2011). Four studies provided details of the content and structure of the home program (Aarts et al., 2012; Abd El-Kafy et al., 2014; Deppe et al., 2013; Eliasson, Shaw, Berg, \& Krumlinde-Sundholm, 2011), three described commercially available video or web-based games (Chiu et al., 2014; James et al., 2015; Zoccolillo et al., 2015) and the remaining studies had inadequate details to replicate either the structure or content of the home program. 
Training for research intervention providers (e.g. workshops) was reported in 17 studies (28\%), however, no study reported adequate details about the content or structure of training, whether there were training materials or where these could be accessed. A further 10 studies $(17 \%)$ reported having procedure manuals, generic or standardized task specific therapy protocols (Charles et al., 2006; Duncan, Shen, Zou, Han, Lu, Zheng et al., 2012; Fehlings, Rang, Glazier, \& Steele, 2000; Gordon et al., 2011; Kawamura, Campbell, LamDamji, \& Fehlings, 2007; Klingels et al., 2013; Olesch, Greaves, Imms, Reid, \& Graham, 2010; Rameckers, Speth, Duysens, Vles, \& Smits-Engelsman, 2009; Speth, Leffers, JanssenPotten, \& Vles, 2005) to guide clinicians in the provision of therapy, however only three provided details that the protocols could be accessed on request (Duncan et al., 2012; Olesch et al., 2010; Sakzewski et al., 2015).

Only one study reported modifications to the intervention during the course of the study (Item 10); with scheduling changes to ensure the anticipated dose of CIMT was provided for children in another study (Deluca, Echols, Law, \& Ramey, 2006). It is not possible to know whether other studies made modifications to aspects of the intervention throughout the duration of the study, but did not report this in the trial report.

\subsection{Control Interventions Compared to Research Interventions}

Compared to the research interventions, description of all items was poorer for the 68 control interventions. The largest differences (20 to 30\%) between the research and control interventions were in descriptions of the intervention rationale, procedures, location of the intervention, and tailoring.

The rationale underpinning the control intervention was adequately described for 40 $(60 \%)$ studies, $33 \%$ less than the research intervention (93\%). The procedure for delivering control interventions was described sufficiently in only $25(37 \%)$ of the studies. Details about whether the control intervention was delivered face to face, individually or in a group was 
described adequately in only $30(44 \%)$ of studies. The best reported item was a brief name of the control intervention (Item 1). Materials were reportedly used in 48 (70\%) control interventions, but adequate details of these were only provided in $10(20 \%)$ studies.

A subgroup of $26(40 \%)$ of the included studies described the control group as receiving "usual care", "standard care" or a similar descriptor. Details on the "usual care" intervention were poor for all items including the rationale or goal underpinning usual care $(31 \%)$, materials $(8 \%)$; procedures $(19 \%)$, intervention provider $(8 \%)$, how the care was delivered (31\%), location (31\%), frequency, duration and length (58\%); tailoring (23\%) and methods and reporting of fidelity $(15 \%)$.

\section{Discussion}

This study highlights the lack of adequate details in reporting research interventions and control interventions in published upper limb rehabilitation trials for children with unilateral cerebral palsy. When using $50 \%$ of studies as the benchmark, five of the 12 TIDieR items for the research intervention, eight of the 12 items for the control intervention and 11 of 12 items for "usual care" interventions were inadequately reported.

Similar to previous findings (Abell, Glasziou, \& Hoffmann, 2015; Bryant, Passey, Hall, \& Sanson-Fisher, 2014; Hoffmann et al., 2013; Pino, Boutron, \& Ravaud, 2012), our results highlight that details about the materials used in the delivery of interventions and in the training of intervention providers were the most poorly reported. Home programs were a key component in many upper limb studies, and one study directly investigated their efficacy (Novak, Cusick, \& Lannin, 2009). Yet, little detail was provided as to what home programs consisted of, their content, how instructions were provided, parent training, the number of activities that were used and how these were developed, individualized and graded. The use of home practice logs by parents was one strategy to measure treatment adherence, yet only one study provided an example (Aarts et al., 2012). Adequate detail and availability of 
intervention materials is a crucial element missing from intervention descriptions and limits reproducibility and implementation of the interventions (Hoffmann et al., 2013). Descriptions of materials need to be in enough detail to allow replication, or otherwise authors need to explicitly state where further details can be sought (e.g. contacting corresponding author).

Reporting of the research intervention setting/location in over half of the trials is similar to previous studies (Abell et al., 2015; Hoffmann et al., 2013). Although tailoring was more highly reported in these paediatric trials of upper limb interventions and reflected individualisation related to collaborative goal setting, the specific details of how tailoring was done and how interventions were progressed was not thoroughly described. Reporting of modifications between the study protocol and duration of the study were absent in all but one study. Authors might consider reporting modification if they were made, or alternatively stating that the intervention delivered as per protocol.

Intervention fidelity was not comprehensively planned, evaluated or reported in most studies. Strategies to optimize and measure treatment fidelity include the training of intervention providers, methods and measurement of delivery and receipt of therapy and enactment of intervention skills (Borrelli, Sepinwall, Ernst, Bellg, Czajkowski, Breger et al., 2005). Training of intervention providers was mentioned in 21 (35\%) studies, however measurement of the provider knowledge and skill acquisition post training was not considered, nor how provider skills were maintained throughout the duration of the study. Intervention in $60 \%$ of included studies was delivered by more than one provider, further reinforcing the need for strategies to monitor and maintain fidelity. The main aspect considered when measuring fidelity in $48 \%$ of trials was recording the intervention dose. No study adequately addressed the aspect of therapist competence. Fidelity is a vital consideration particularly for complex interventions included in the current study. Reporting fidelity aids in interpretation of results and understanding the essential components of 
complex interventions. The lack of methodological strategies to enhance and monitor delivery of research interventions decreases confidence in both the internal and external validity of the studies (Borrelli et al., 2005).

To our knowledge, this is the first study that has analysed the completeness of descriptions of control interventions in randomized trials. Inadequacy of reporting was even greater when the comparison to a research intervention was "usual care". "Usual care" is likely very different between studies and highly dependent on factors such as the clinician involved, clinical setting and country in which the care is provided. Hence this lack of detail limits accurate interpretation of the magnitude of treatment effects of the research intervention being evaluated, and hampers comparison of effect sizes across studies.

A number of factors contribute to the lack of adequate description of interventions in randomized trials. A recent cross sectional study investigated the extent to which journals' instructions to authors provided adequate details on how to report interventions and whether they allowed supplementary online materials to be published (Hoffmann, English, \& Glasziou, 2014a). Across 106 journals, only 14\% mentioned intervention reporting, although most gave non-specific instructions such as "describe methods, including interventions"; $58 \%$ referred to the CONSORT statement; and whilst $74 \%$ of journals offered supplementary online options, only 4\% mandated their use (Hoffmann et al., 2014a). Word/page restrictions in journals challenge authors' abilities to furnish adequate details on the interventions in the published report (Glasziou, Chalmers, Altman, Bastian, Boutron, Brice et al., 2010). However, greater use of the supplementary online options offered by journals can provide authors with additional scope to report interventions in greater detail. Another contributing factor is the lack of awareness of authors, reviewers and editors on the importance of complete reporting of interventions (Schulz, Altman, \& Moher, 2010). Development of the TIDieR checklist (Hoffmann et al., 2014b), if adopted consistently by journals, editors and 
authors of protocols and trials, has the potential to improve the quality of reporting of intervention and control conditions.

A strength of this study is the evaluation of the description of not only the research intervention, but also the control intervention. Additionally, this study included all randomised trials of upper limb therapy irrespective of publication year (Hoffmann et al., 2013) or journal quality (Glasziou et al., 2008). Unlike previous studies (Abell et al., 2015; Hoffmann et al., 2013), however, we did not approach authors to gain additional information to determine whether the completeness of interventions details could be improved. Previous studies have shown that additional information can be gained directly from authors (Hoffmann et al., 2013) and researchers and clinicians should contact authors to determine if there is more detailed information available to assist with knowledge translation processes. Additionally, we only included randomised controlled trials in the current study, however, the TIDieR checklist should would be a valuable tool to assist with reporting of interventions using other research designs.

\section{Conclusion}

Few research interventions of upper limb therapies for children with unilateral cerebral palsy were described with sufficient detail to enable replication of the intervention, with crucial details missing in many. Even poorer reporting was observed for control interventions. Lack of comprehensive reporting of interventions contributes to a worldwide waste in research funding, limits the uptake of research findings in clinical practice, and hampers evidence synthesis. Authors, reviewers, and editors all share a responsibility for improving the quality of intervention reporting in published trials. Mandating use of the TIDieR checklist and guide in reporting interventions is a potential solution for making it easier to structure accounts of intervention. 
Acknowledgements, funding and support: We wish to thank Mr Vineel Lal who assisted with rating 54 of the included papers and was supported by a University of Queensland Summer Student Scholarship; National Health and Medical Research Council of Australia Early Career Research Fellowship (LS: 1090828). 


\section{References}

Aarts, P. B., van Hartingsveldt, M., Anderson, P. G., van den Tillaar, I., van der Burg, J., \& Geurts, A. C. (2012). The Pirate Group Intervention Protocol: Description and a Case Report of a Modified Constraint-induced Movement Therapy Combined with Bimanual Training for Young Children with Unilateral Spastic Cerebral Palsy. Occupational Therapy International, 19(2), 76-87. doi: 10.1002/oti.321

Abd El-Kafy, E. M., Elshemy, S. A., \& Alghamdi, M. S. (2014). Effect of constraint-induced therapy on upper limb functions: A randomized control trial. Scandinavian Journal of Occupational Therapy, 21(1), 11-23. doi: 10.3109/11038128.2013.837505

Abell, B., Glasziou, P., \& Hoffmann, T. (2015). Reporting and replicating trials of exercise-based cardiac rehabilitation: do we know what the researchers actually did? Circulation: Cardiovascular Quality and Outcomes, 8(2), 187-194. doi: 10.1161/circoutcomes.114.001381

Bleyenheuft, Y., Arnould, C., Brandao, M. B., Bleyenheuft, C., \& Gordon, A. M. (2014). Hand and Arm Bimanual Intensive Therapy Including Lower Extremity (HABIT-ILE) in Children With Unilateral Spastic Cerebral Palsy: A Randomized Trial. Neurorehabilitation and Neural Repair, 29(7), 645-657. doi: 10.1177/1545968314562109

Bleyenheuft, Y., \& Gordon, A. M. (2014). Hand-Arm Bimanual Intensive Therapy Including Lower Extremities (HABIT-ILE) for Children with Cerebral Palsy. Physical \& Occupational Therapy in Pediatrics, 34(4), 390-403. doi: 10.3109/01942638.2014.932884

Borrelli, B., Sepinwall, D., Ernst, D., Bellg, A. J., Czajkowski, S., Breger, R., et al. (2005). A new tool to assess treatment fidelity and evaluation of treatment fidelity across 10 years of health behavior research. Journal of Consulting and Clinical Psychology, 73(5), 852-860. doi: 10.1037/0022-006X.73.5.852

Boyd, R., Sakzewski, L., Ziviani, J., Abbott, D. F., Badawy, R., Gilmore, R., et al. (2010). INCITE: A randomised trial comparing constraint induced movement therapy and bimanual training in children with congenital hemiplegia. BMC Neurology, 10.

Boyd, R. N., Mitchell, L. E., James, S. T., Ziviani, J., Sakzewski, L., Smith, A., et al. (2013). Move it to improve it (Mitii): study protocol of a randomised controlled trial of a novel web-based multimodal training program for children and adolescents with cerebral palsy. BMJ Open, 3(4). doi: 10.1136/bmjopen-2013-002853

Boyd, R. N., Ziviani, J., Sakzewski, L., Miller, L., Bowden, J., Cunnington, R., et al. (2013). COMBIT: protocol of a randomised comparison trial of COMbined modified constraint induced movement therapy and bimanual intensive training with distributed model of standard upper limb rehabilitation in children with congenital hemiplegia. BMC Neurology, 13, 68. doi: 10.1186/1471-2377-13-68

Brandao, M. B., Ferre, C., Kuo, H. C., Rameckers, E. A., Bleyenheuft, Y., Hung, Y. C., et al. (2013). Comparison of Structured Skill and Unstructured Practice During Intensive Bimanual Training in Children With Unilateral Spastic Cerebral Palsy. Neurorehabilitation and Neural Repair, 28(5), 452-461. doi: 10.1177/1545968313516871

Bryant, J., Passey, M. E., Hall, A. E., \& Sanson-Fisher, R. W. (2014). A systematic review of the quality of reporting in published smoking cessation trials for pregnant women: an explanation for the evidence-practice gap? Implementation Science, 9, 94. doi: 10.1186/s13012-014-0094-z

Chan, A. W., Tetzlaff, J. M., Altman, D. G., Laupacis, A., Gotzsche, P. C., Krleza-Jeric, K., et al. (2013). SPIRIT 2013 Statement: Defining Standard Protocol Items for Clinical Trials. Annals of Internal Medicine, 158(3), 200-+.

Charles, J., \& Gordon, A. (2006). Development of hand-arm bimanual intensive training (HABIT) for improving bimanual coordination in children with hemiplegic cerebral palsy. Developmental Medicine \& Child Neurology, 48(11), 931-936. 
Charles, J., Wolf, S., Schneider, J., \& Gordon, A. (2006). Efficacy of a child-friendly form of constraintinduced movement therapy in hemiplegic cerebral palsy: a randomized control trial. Developmental Medicine \& Child Neurology, 48(8), 635-642.

Chiu, H. C., Ada, L., \& Lee, H. M. (2014). Upper limb training using Wii Sports Resort for children with hemiplegic cerebral palsy: a randomized, single-blind trial. Clinical Rehabilitation, 28(10), 1015-1024. doi: 10.1177/0269215514533709

Deluca, S. C., Echols, K., Law, C. R., \& Ramey, S. L. (2006). Intensive pediatric constraint-induced therapy for children with cerebral palsy: Randomized, controlled, crossover trial. Journal of Child Neurology, 21(11), 931-938. doi: 10.2310/7010.2006.00201

Deppe, W., Thuemmler, K., Fleischer, J., Berger, C., Meyer, S., \& Wiedemann, B. (2013). Modified constraint-induced movement therapy versus intensive bimanual training for children with hemiplegia - a randomized controlled trial. Clinical Rehabilitation, 27(10), 909-920. doi: $10.1177 / 0269215513483764$

Dong, A. V., \& Fong, N. K. (2014). Remind to move - A novel treatment on hemiplegic arm functions in children with unilateral cerebral palsy: A randomized cross-over study. Developmental Neurorehabilitation, 1-9. doi: 10.3109/17518423.2014.988304

Douet, L., Milne, R., Anstee, S., Habens, F., Young, A., \& Wright, D. (2014). The completeness of intervention descriptions in published National Institute of Health Research HTA-funded trials: a cross-sectional study. BMJ Open, 4(1), e003713. doi: 10.1136/bmjopen-2013-003713

Duncan, B., Shen, K. L., Zou, L. P., Han, T. L., Lu, Z. L., Zheng, H., et al. (2012). Evaluating intense rehabilitative therapies with and without acupuncture for children with cerebral palsy: A randomized controlled trial. Archives of Physical Medicine and Rehabilitation, 93(5), 808815. doi: 10.1016/j.apmr.2011.12.009

Eliasson, A. C., Krumlinde-Sundholm, L., Shaw, K., \& Wang, C. (2005). Effects of constraint-induced movement therapy in young children with hemiplegic cerebral palsy: an adapted model. Developmental Medicine \& Child Neurology, 47(4), 266-275.

Eliasson, A. C., Shaw, K., Berg, E., \& Krumlinde-Sundholm, L. (2011). An ecological approach of Constraint Induced Movement Therapy for 2-3-year-old children: A randomized control trial. Research in Developmental Disabilities, 32(6), 2820-2828. doi: 10.1016/j.ridd.2011.05.024

Elvrum, A., Braendvik, S., Saether, R., Lamvik, T., Vereijken, B., \& Roeleveld, K. (2012). Effectiveness of resistance training in combination with botulinum toxin-A on hand and arm use in children with cerebral palsy: A pre-post intervention study. Bmc Pediatrics, 12, 91. doi:10.1186/1471-2431-12-91.

Facchin, P., Rosa-Rizzotto, M., Turconi, A., Pagliano, E., Fazzi, E., Stortini, M., et al. (2009). Multisite trial on efficacy of constraint-induced movement therapy in children with hemiplegia. American Journal of Physical Medicine and Rehabilitation, 88(3), 216-230.

Fehlings, D., Rang, M., Glazier, J., \& Steele, C. (2000). An evaluation of botulinum-A toxin injections to improve upper extremity function in children with hemiplegic cerebral palsy. Journal of Pediatrics, 137(3), 331-337.

Ferrari, A., Maoret, A. R., Muzzini, S., Alboresi, S., Lombardi, F., Sgandurra, G., et al. (2014). A randomized trial of upper limb botulimun toxin versus placebo injection, combined with physiotherapy, in children with hemiplegia. Research in Developmental Disabilities, 35(10), 2505-2513. doi: 10.1016/j.ridd.2014.06.001

Gelkop, N., Burshtein, D. G., Lahav, A., Brezner, A., Al-Oraibi, S., Ferre, C. L., et al. (2014). Efficacy of Constraint-Induced Movement Therapy and Bimanual Training in Children with Hemiplegic Cerebral Palsy in an Educational Setting. Physical \& Occupational Therapy in Pediatrics. doi: 10.3109/01942638.2014.925027

Gilliaux, M., Renders, A., Dispa, D., Holvoet, D., Sapin, J., Dehez, B., et al. (2015). Upper limb robotassisted therapy in cerebral palsy: a single-blind randomized controlled trial. Neurorehabilitation and Neural Repair, 29(2), 183-192. doi: 10.1177/1545968314541172 
Glasziou, P., Altman, D. G., Bossuyt, P., Boutron, I., Clarke, M., Julious, S., et al. (2014). Reducing waste from incomplete or unusable reports of biomedical research. Lancet, 383(9913), 267276. doi: 10.1016/s0140-6736(13)62228-x

Glasziou, P., Chalmers, I., Altman, D. G., Bastian, H., Boutron, I., Brice, A., et al. (2010). Taking healthcare interventions from trial to practice. $B M J, 341$, c3852. doi: 10.1136/bmj.c3852

Glasziou, P., Meats, E., Heneghan, C., \& Shepperd, S. (2008). What is missing from descriptions of treatment in trials and reviews? British Medical Journal, 336(7659), 1472-1474. doi: 10.1136/bmj.39590.732037.47

Gordon, A., Schneider, J., Chinnan, A., \& Charles, J. (2007). Efficacy of a hand-arm bimanual intensive therapy (HABIT) in children with hemiplegic cerebral palsy: a randomized control trial. Developmental Medicine and Child Neurology, 49(11), 830-838.

Gordon, A. A., Charles, J., \& Wolf, S. L. (2005). Methods of constraint-induced movement therapy for children with hemiplegic cerebral palsy: Development of a child-friendly intervention for improving upper-extremity function. Archives of Physical Medicine \& Rehabilitation, 86(4), 837-844. doi: 10.1016/j.apmr.2004.10.008

Gordon, A. M., Hung, Y. C., Brandao, M., Ferre, C. L., Kuo, H. C., Friel, K., et al. (2011). Bimanual training and constraint-induced movement therapy in children with hemiplegic cerebral palsy: A randomized trial. Neurorehabilitation \& Neural Repair, 25(8), 692-702. doi: 10.1177/1545968311402508

Hoare, B. J., Imms, C., Rawicki, H. B., \& Carey, L. (2010). Modified constraint-induced movement therapy or bimanual occupational therapy following injection of Botulinum toxin-A to improve bimanual performance in young children with hemiplegic cerebral palsy: $A$ randomised controlled trial methods paper. BMC Neurology, 10, 58.

Hoffmann, T., English, T., \& Glasziou, P. (2014a). Reporting of interventions in randomised trials: an audit of journal instructions to authors. Trials, 15, 20. doi: 10.1186/1745-6215-15-20

Hoffmann, T., Erueti, C., \& Glasziou, P. (2013). Poor description of non-pharmacological interventions: analysis of consecutive sample of randomised trials. British Medical Journal, 347, f3755. doi: 10.1136/bmj.f3755

Hoffmann, T. C., Glasziou, P. P., Boutron, I., Milne, R., Perera, R., Moher, D., et al. (2014b). Better reporting of interventions: template for intervention description and replication (TIDieR) checklist and guide. British Medical Journal, 348. doi: 10.1136/bmj.g1687

Hsin, Y. J., Chen, F. C., Lin, K. C., Kang, L. J., Chen, C. L., \& Chen, C. Y. (2012). Efficacy of constraintinduced therapy on functional performance and health-related quality of life for children with cerebral palsy: A randomized controlled trial. Journal of Child Neurology, 27(8), 992999. doi: $10.1177 / 0883073811431011$

James, S., Ziviani, J., Ware, R. S., \& Boyd, R. N. (2015). Randomized controlled trial of web-based multimodal therapy for unilateral cerebral palsy to improve occupational performance. Developmental Medicine \& Child Neurology, 57(6), 530-538. doi: 10.1111/dmcn.12705

Kawamura, A., Campbell, K., Lam-Damji, S., \& Fehlings, D. (2007). A randomized controlled trial comparing botulinum toxin $A$ dosage in the upper extremity of children with spasticity. Developmental Medicine \& Child Neurology, 49(5), 331-337.

Kaya Kara, O., Atasavun Uysal, S., Turker, D., Karayazgan, S., Gunel, M. K., \& Baltaci, G. (2015). The effects of Kinesio Taping on body functions and activity in unilateral spastic cerebral palsy: a single-blind randomized controlled trial. Developmental Medicine \& Child Neurology, 57(1), 81-88. doi: 10.1111/dmcn.12583

Klingels, K., Feys, H., Molenaers, G., Verbeke, G., Van Daele, S., Hoskens, J., et al. (2013). Randomized Trial of Modified Constraint-Induced Movement Therapy With and Without an Intensive Therapy Program in Children With Unilateral Cerebral Palsy. Neurorehabilitation \& Neural Repair, 27(9), 799-807. doi: 10.1177/1545968313496322

Koman, L. A., Smith, B. P., Williams, R., Richardson, R., Naughton, M., Griffin, L., et al. (2013). Upper extremity spasticity in children with cerebral palsy: a randomized, double-blind, placebo- 
controlled study of the short-term outcomes of treatment with botulinum A toxin. The Journal of hand surgery, 38(3), 435-446.e431. doi: 10.1016/j.jhsa.2012.12.019

Law, M., Cadman, D., Rosenbaum, P., Walter, S., Russell, D., \& Dematteo, C. (1991). Neurodevelopmental therapy and upper-extremity inhibitive casting for children with cerebral palsy. Developmental Medicine \& Child Neurology, 33(5), 379-387.

Law, M., Darrah, J., Pollock, N., Rosenbaum, P., Russell, D., Walter, S. D., et al. (2007). Focus on Function - a randomized controlled trial comparing two rehabilitation interventions for young children with cerebral palsy. Bmc Pediatrics, 7, 31.

Law, M., Russell, D., Pollock, N., Rosenbaum, P., Walter, S., \& King, G. (1997). A comparison of intensive neurodevelopmental therapy plus casting and a regular occupational therapy program for children with cerebral palsy. Developmental Medicine \& Child Neurology, 39(10), 664-670.

Lidman, G., Nachemson, A., Peny-Dahlstrand, M., \& Himmelmann, K. (2015). Botulinum toxin A injections and occupational therapy in children with unilateral spastic cerebral palsy: a randomized controlled trial. Developmental Medicine \& Child Neurology. doi: $10.1111 /$ dmcn.12739

McConnell, K., Johnston, L., \& Kerr, C. (2012). Therapy management of the upper limb in children with cerebral palsy: a cross-sectional survey. Developmental Neurorehabilitation, 15(5), 343350. doi: 10.3109/17518423.2012.692124

Moher, D., Hopewell, S., Schulz, K. F., Montori, V., Gotzsche, P. C., Devereaux, P. J., et al. (2010). CONSORT 2010 Explanation and Elaboration: Updated guidelines for reporting parallel group randomised trials. Journal of Clinical Epidemiology, 63(8), e1-37. doi: 10.1016/j.jclinepi.2010.03.004

Novak, l., Cusick, A., \& Lannin, N. (2009). Occupational therapy home programs for cerebral palsy: Double-blind, randomized, controlled trial. Pediatrics, 124(4), e606-e614.

Novak, I., Cusick, A., \& Lowe, K. (2007). A pilot study on the impact of occupational therapy home programming for young children with cerebral palsy. American Journal of Occupational Therapy, 61(4), 463-468.

Novak, I., McIntyre, S., Morgan, C., Campbell, L., Dark, L., Morton, N., et al. (2013). A systematic review of interventions for children with cerebral palsy: state of the evidence. Developmental Medicine \& Child Neurology, 55(10), 885-910. doi: 10.1111/dmcn.12246

Olesch, C., Greaves, S., Imms, C., Reid, S., \& Graham, H. (2010). Repeat botulinum toxin-A injections in the upper limb of children with hemiplegia: a randomized controlled trial. Dev Med Child Neurol., 52(1), 79-86.

Pino, C., Boutron, I., \& Ravaud, P. (2012). Inadequate description of educational interventions in ongoing randomized controlled trials. Trials, 13, 63. doi: 10.1186/1745-6215-13-63

Rameckers, E., Speth, L., Duysens, J., Vles, J., \& Smits-Engelsman, B. (2009). Botulinum toxin-A in children with congenital spastic hemiplegia does not improve upper extremity motor-related function over rehabilitation alone: a randomized controlled trial. Neurorehabilitation \& Neural Repair, 23(3), 218-225. doi: 10.1177/1545968308326629

Sakzewski, L., Miller, L., Ziviani, J., Abbott, D. F., Rose, S., Macdonell, R. A., et al. (2015). Randomized comparison trial of density and context of upper limb intensive group versus individualized occupational therapy for children with unilateral cerebral palsy. Developmental Medicine \& Child Neurology, 57(6), 539-547. doi: 10.1111/dmcn.12702

Sakzewski, L., Ziviani, J., \& Boyd, R. N. (2014). Efficacy of upper limb therapies for unilateral cerebral palsy: a meta-analysis. Pediatrics, 133(1), e175-204. doi: 10.1542/peds.2013-0675

Schertz, M., \& Gordon, A. (2008). Changing the model: a call for a re-examination of intervention approaches and translational research in children with developmental disabilities. Developmental Medicine \& Child Neurology, 51(1), 6-7.

Schroter, S., Glasziou, P., \& Heneghan, C. (2012). Quality of descriptions of treatments: a review of published randomised controlled trials. BMJ Open, 2(6). doi: 10.1136/bmjopen-2012-001978 
Schulz, K., Altman, D., \& Moher, D. (2010). CONSORT 2010 Statement: updated guidelines for reporting parallel group randomised trials. $B M C$ Med, $8,18$.

Sgandurra, G., Ferrari, A., Cossu, G., Guzzetta, A., Fogassi, L., \& Cioni, G. (2013). Randomized trial of observation and execution of upper extremity actions versus action alone in children with unilateral cerebral palsy. Neurorehabilitation and Neural Repair, 27(9), 808-815. doi: $10.1177 / 1545968313497101$

Sgandurra, G., Sicola, E., Di Pietro, R., Burzi, V., Filippi, M. C., Parente, E., et al. (2011). Upper limb children action-observation training (UP-CAT) study: Design of a randomised clinical trial for children with hemiplegia. Developmental Medicine \& Child Neurology, 53, 56.

Speth, L., Leffers, P., Janssen-Potten, Y. J. M., \& Vles, J. S. H. (2005). Botulinum toxin A and upper limb functional skills in hemiparetic cerebral palsy: A randomized trial in children receiving intensive therapy. Dev Med Child Neurol., 47(7), 468-473.

Taub, E., Griffin, A., Uswatte, G., Gammons, K., Nick, J., \& Law, C. R. (2011). Treatment of congenital hemiparesis with pediatric constraint-induced movement therapy. Journal of Child Neurology, 26(9), 1163-1173. doi: 10.1177/0883073811408423

Wallen, M., Ziviani, J., Herbert, R., Evans, R., \& Novak, I. (2009). Modified constraint-induced therapy for children with hemiplegic cerebral palsy: A feasibility study. Developmental Neurorehabilitation, 11(2), 124-133.

Wallen, M. A., O'Flaherty S, J., \& Waugh, M. C. (2004). Functional outcomes of intramuscular botulinum toxin type $A$ in the upper limbs of children with cerebral palsy: a phase II trial. Archives of Physical Medicine \& Rehabilitation, 85(2), 192-200.

Yu, J., Kang, H., \& Jung, J. (2012). Effects of Modified Constraint-induced Movement Therapy on Hand Dexterity, Grip Strength and Activities of Daily Living of Children with Cerebral Palsy: a Randomized Control Trial. Journal of Physical Therapy Science, 24(10), 1029-1031.

Zoccolillo, L., Morelli, D., Cincotti, F., Muzzioli, L., Gobbetti, T., Paolucci, S., et al. (2015). Video-game based therapy performed by children with cerebral palsy: a cross-over randomized controlled trial and a cross-sectional quantitative measure of physical activity. European Journal of Physical and Rehabilitation Medicine. 
Figure 1. Percentage of adequate reporting of research interventions, comparison interventions and usual care according to the TIDieR Checklist.

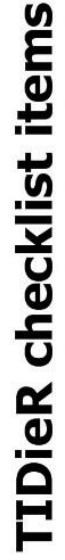

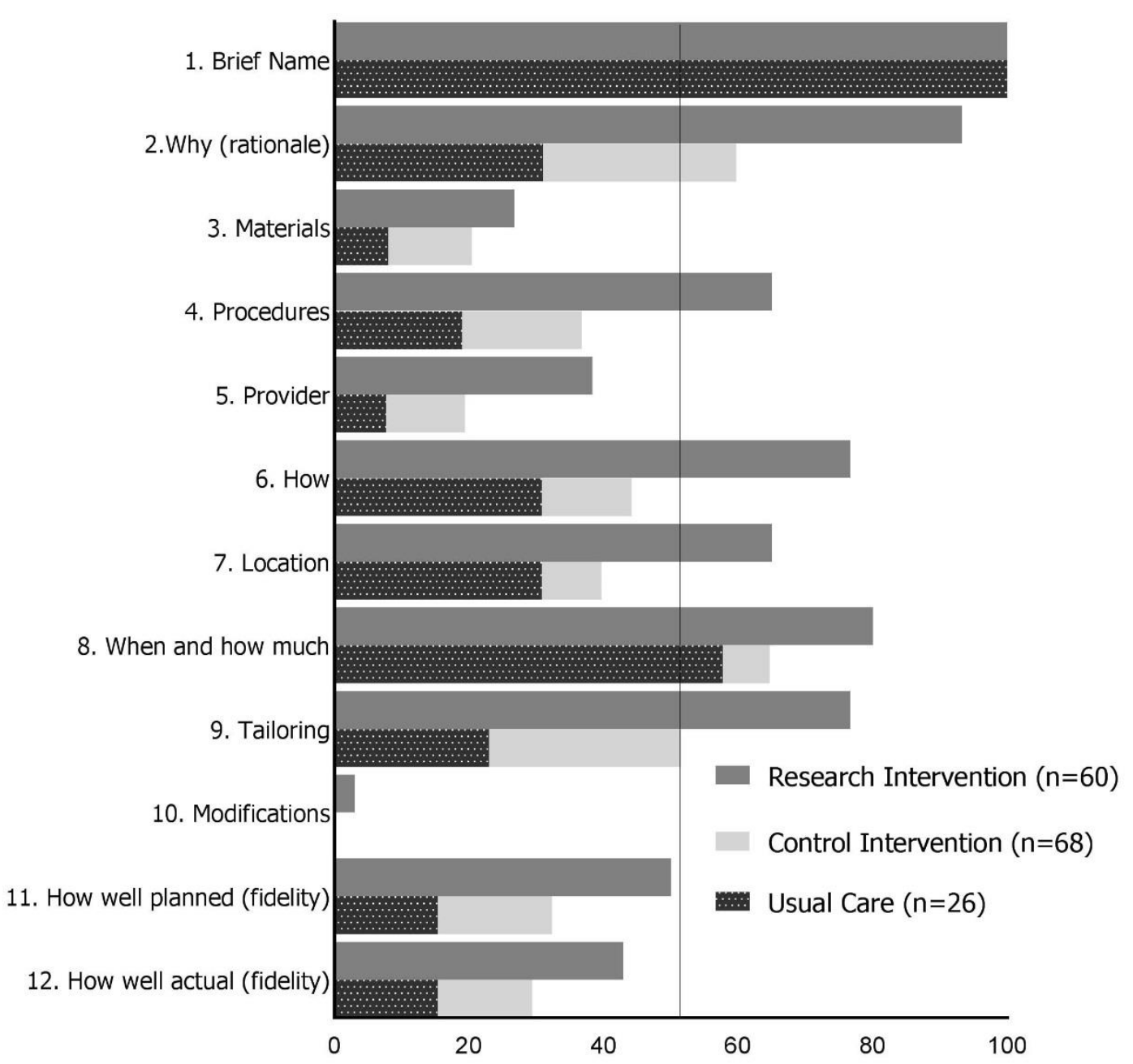

$\%$ of studies with adequate reporting 
Table 1. Description of TIDieR items and examples of adequate reporting in included studies

\begin{tabular}{|c|c|c|}
\hline TIDieR Item & Item description & Examples of adequate reporting \\
\hline 1. Brief Name & $\begin{array}{l}\text { Provide the name or a phrase } \\
\text { that describes the intervention. }\end{array}$ & $\begin{array}{l}\text { "Modified constraint induced movement therapy (mCIMT)" (Hoare, Imms, Villanueva, } \\
\text { Rawicki, Matyas, \& Carey, 2013; Klingels et al., 2013; Wallen, Ziviani, Naylor, Evans, Novak, \& } \\
\text { Herbert, 2011). }\end{array}$ \\
\hline 2. Why & $\begin{array}{l}\text { Describe any rationale, theory, } \\
\text { or goal of the elements } \\
\text { essential to the intervention. }\end{array}$ & $\begin{array}{l}\text { "...both imagery and actual execution of hand actions activate similar structures of the } \\
\text { sensorimotor cortex....action observation, combined with actual replication of the observed } \\
\text { action, induces a strong activation of the MNS, along with marked improvement in motor } \\
\text { learning efficacy. These results have fostered the development of rehabilitation protocols } \\
\text { based on the observation of meaningful actions followed by their execution (Observation to } \\
\text { Imitate)." [Action Observation Training (Sgandurra et al., 2013)]. }\end{array}$ \\
\hline 3. What: Materials & $\begin{array}{l}\text { Describe any physical or } \\
\text { informational materials used in } \\
\text { the intervention, including } \\
\text { those provided to participants } \\
\text { or used in the intervention } \\
\text { delivery or training of } \\
\text { intervention providers. Provide } \\
\text { information on where the } \\
\text { materials can be accessed. }\end{array}$ & $\begin{array}{l}\text { "Most treatment was undertaken with the child sitting at a height adjustable table (see } \\
\text { Figure 1)...The child's chair, with footrest, armrest and pommel was adjusted so that the } \\
\text { table was at waist height." [mCIMT and BoNT-A (Hoare et al., 2010)]. }\end{array}$ \\
\hline 4. What: Procedures & $\begin{array}{l}\text { Describe each of the } \\
\text { procedures, activities, and/or } \\
\text { processes used in the } \\
\text { intervention, including any } \\
\text { enabling or support activities. }\end{array}$ & $\begin{array}{l}\text { "After baseline assessment, a trained therapist casted the child's stronger or non-involved } \\
\text { arm from the axillary area to the end of the fingertips with the elbow positioned in } 90^{\circ} \\
\text { flexion.....The therapist removed the cast once a week during the intervention period to check } \\
\text { skin integrity and allow the child } 15-20 \text { min of active range of motion.... The therapist } \\
\text { structured the practice of arm and hand movements into activities of daily living (e.g., } \\
\text { dressing and undressing, eating, grooming) and play activities....At the end of the }\end{array}$ \\
\hline
\end{tabular}
intervention Day 18, the therapist removed the cast and shifted the focus of the intervention 
5. Who Provided

6. How

7. Where

8. When and How Much
For each category of intervention provider (e.g. psychologist, nursing assistant), describe their expertise, background and any specific training given.

Describe the modes of delivery (e.g. face to face or by some other mechanism, such as internet or telephone) of the intervention and whether it was provided individually or in a group.

Describe the type(s) of location(s) where the intervention occurred, including any necessary infrastructure or relevant features.

Describe the number of times the intervention was delivered and over what period of time including the number of sessions, their schedule, and their duration, intensity of dose. for the final 3 days to bimanual activities" [CIMT (Case-Smith, DeLuca, Stevenson, \& Ramey, 2012)].

"Three occupational therapists and one physiotherapist will plan and lead all intervention groups.... Volunteer occupational therapists, physiotherapists, human movement scientists, therapy students and sports recreation staff (YMCA) will assist with program delivery with a ratio of 2 participants to one staff member....Professional circus trainers will lead the two hour circus workshops" [INCITE- mCIMT (Boyd et al., 2010)].

"The pretreatment training, administered by the supervisors, was standardized based on the established manual of procedures for each treatment and reinforced by supervisors and during daily meetings." [mCIMT and HABIT (Gordon et al., 2011)].

"Participants worked individually with their interventionist or in groups (1:1 interventionist to participant ratio always maintained). Interventionists were paired with children prior to randomization using family-centered approaches considering caregiver and supervisors' best judgement based on the child's age and gender" [mCIMT and HABIT (Gordon et al., 2011)].

"The intervention ...during which participating children visit the out-patient rehabilitation unit of the Sint Maartenskliniek, Nijmegen, the Netherlands .....The rooms for the intervention are decorated as a pirate island with all kinds of pirate attributes." [The Pirate Group: hybrid-CIMT (Aarts et al., 2012)].

"The individual-based treatment sessions of 45 to 60 minutes were conducted ...twice weekly for 8 weeks in an outpatient paediatric treatment room. In addition, children in the mCIMT experimental group were required to complete 3 hours of home program (with mitt on), 7 days a week for the 8 week treatment period" [mCIMT and BoNT-A (Hoare et al., 2010)]. 
9. Tailoring

10. Modifications

11. How Well Planned

12. How Well Actual
If the intervention was planned to be personalised, titrated or adapted, then describe what, why, when and how.

If the intervention was modified during the course of the study, describe the changes (what, why, when and how).

If intervention adherence or fidelity was assessed, describe how and by whom, and if any strategies were used to maintain or improve fidelity, describe them.

If intervention adherence or fidelity was assessed, describe the extent to which the intervention was delivered as planned.
"Task difficulty was graded as the child's performance improved by requiring greater speed or accuracy, or by providing tasks that required more skilled use of the involved hand and arm (e.g. moving from activities in which the involved limb acted as a stabilizer to activities that required manipulative skills). Interventionists altered constraints to grade tasks according to desired target movements (e.g. they built up the grasp surface of an object by adding tape and removed it as grasp improved). Emphasis was placed on completing each movement with the involved upper extremity in the same way as the non-dominant hand of a typically developing child (i.e. as a stabilizer or manipulator)." [HABIT) (Gordon et al., 2007)].

"When children took naps or had an unexpected disruption of their treatment, the therapist was responsible for ensuring that the full dose of 6 hours of active treatment per day was provided (e.g. by staying longer that day or by scheduling treatment for 3 hours before naptime and 3 hours after naptime)" [signature CIMT (Deluca et al., 2006)].

"A clearly defined classification of intervention strategies was developed for each intervention approach to unsure that they were indeed different.... To measure therapist adherence, children's attendance was monitored throughout the study. All therapists completed a log after each session to document therapy, and these were analysed to determine procedural reliability" [Context-focused intervention (Law, Darrah, Pollock, Wilson, Russell, Walter et al., 2011)].

"Therapist log notes were coded to record the five most frequent intervention strategies in order to explore treatment differentiation between groups. In the child-focused group, they were practice of upper extremity motor activities........ In the context-focused group they were modifying physical characteristics of environment....... The most frequent intervention strategies were distinct for each group except for the practice of functional mobility activities." [Context-focused intervention (Law et al., 2011)]. 
Table 2. Categories of interventions ( $n=60$ research interventions published in 71 articles, 3 feasibility studies, and 12 protocols) and publication sources of included studies

\begin{tabular}{|c|c|c|c|c|}
\hline Category of intervention & $\begin{array}{l}\text { Number and proportion } \\
\text { of published studies for } \\
\text { each intervention } \\
\mathrm{N}(\%)\end{array}$ & $\begin{array}{l}\text { Number of published } \\
\text { feasibility studies, } \\
\text { protocols, supporting } \\
\text { articles for the } \\
\text { intervention }\end{array}$ & $\begin{array}{l}\text { Category of } \\
\text { control/comparison } \\
\text { interventions }\end{array}$ & $\begin{array}{l}\text { Number of } \\
\text { control/comparison } \\
\text { interventions } \mathrm{N}\end{array}$ \\
\hline Constraint induced movement therapy & $26(43)$ & 8 & $\begin{array}{l}\text { Bimanual therapy/OT/PT } \\
\text { Usual Care } \\
\text { NDT } \\
\text { Control } \\
\text { CIMT (diff dose/context) }\end{array}$ & $\begin{array}{l}9 \\
10 \\
1 \\
4 \\
5\end{array}$ \\
\hline $\begin{array}{l}\text { Botulinum Toxin A and occupational therapy } \pm \\
\text { splint, FES }\end{array}$ & $14(23)$ & 1 & $\begin{array}{l}\text { Control } \\
\text { OT/PT } \\
\text { Placebo } \pm \text { PT } \\
\text { BoNT-A (diff dose) } \\
\text { BoNT-A + OT }\end{array}$ & $\begin{array}{l}1 \\
8 \\
2 \\
1 \\
3\end{array}$ \\
\hline Virtual reality/robotics & $4(6)$ & 1 & Usual care & 4 \\
\hline Neurodevelopmental Therapy + casting & $2(3)$ & 0 & $\begin{array}{l}\text { Intensive NDT } \\
\text { Regular NDT } \\
\text { OT }\end{array}$ & $\begin{array}{l}1 \\
1 \\
1\end{array}$ \\
\hline Forced use therapy & $2(3)$ & 0 & $\begin{array}{l}\text { Usual Care } \\
\text { Control }\end{array}$ & $\begin{array}{l}1 \\
1\end{array}$ \\
\hline Action observation training & $2(3)$ & 1 & Watching other video & 1 \\
\hline $\begin{array}{l}\text { Hand-Arm Bimanual Intensive Training ( } \pm \\
\text { intensive LE) }\end{array}$ & $3(5)$ & 2 & $\begin{array}{l}\text { Control } \\
\text { Usual Care } \\
\text { Unstructured bimanual }\end{array}$ & $\begin{array}{l}1 \\
1 \\
1\end{array}$ \\
\hline
\end{tabular}




\begin{tabular}{|c|c|c|c|c|}
\hline Mirror therapy & $1(2)$ & 0 & Bimanual therapy & 1 \\
\hline \multirow[t]{2}{*}{ Occupational therapy home programs } & $1(2)$ & 1 & Home program (diff dose) & 1 \\
\hline & & & Control & 1 \\
\hline Acupuncture + occupational therapy & $1(2)$ & 0 & Intensive therapy & 1 \\
\hline Context-focused intervention & $1(2)$ & 1 & Child focused therapy & 1 \\
\hline Splinting & $1(2)$ & 0 & Goal directed training & 1 \\
\hline Sensory cuing & $1(2)$ & 0 & Sham & 1 \\
\hline Kinesiotape & $1(2)$ & 0 & Usual care & 1 \\
\hline
\end{tabular}

Diff, different; OT, occupational therapy; PT, physiotherapy; NDT, neurodevelopmental therapy; BoNT-A, Botulinum Toxin A; LE, lower extremity; FES, functional electrical stimulation. 